\title{
Fractional calculus pertaining to multivariable $I$-function defined by Prathima
}

\author{
D. KUMAR AND F. Y. AYANT
}

\begin{abstract}
In this paper, we study a pair of unified and extended fractional integral operator involving the multivariable $I$-functions and general class of multivariable polynomials. Here, we use Mellin transforms to obtain our main results. Certain properties of these operators concerning to their Mellin-transforms have been investigated. On account of the general nature of the functions involved herein, a large number of known (may be new also) fractional integral operators involved simpler functions can be obtained. We will also quote the particular case of the multivariable $H$-function.
\end{abstract}

Mathematics Subject Classification 2010: Primary 33C45, 33C60; Secondary 26D20.

Keywords: Multivariable $I$-function; $I$-function; Fractional integral operators; General class of multivariable polynomials; Mellin transform; Multivariable $H$-function.

\section{INTRODUCTION AND PRELIMINARIES}

Fractional calculus is a field of applied mathematics that deals with derivatives and integrals of arbitrary orders. Recently, it has turned out many phenomena in physics, mechanics, chemistry, biology and other sciences can be described very successfully by models using mathematical tools by models using mathematical tools from fractional calculus. Chaurasia and Srivastava [4], Choi et al. [5], Daiya et al. [6], Kumar and Daiya [10], Kumar et al. [12], and others have studied the fractional calculus pertaining to multivariable $H$-function defined by Srivastava and Panda [24].

The $\bar{I}$ - function, introduced by Rathie [16], however the notation and complete definition is presented here in the following manner in terms of the Mellin-Barnes type integral:

$$
\bar{I}(z)=\bar{I}_{p, q}^{m, n}\left[z \mid \begin{array}{c}
\left(a_{j}, \alpha_{i} ; A_{j}\right)_{n, n+1},\left(a_{j}, \alpha_{i} ; A_{j}\right)_{p} \\
\left(b_{j}, \beta_{j} ; B_{j}\right)_{m, m+1},\left(b_{j}, \beta_{j} ; B_{j}\right)_{q}
\end{array}\right]=\frac{1}{2 \pi \omega} \int_{L} \Omega_{p, q}^{m, n}(s) z^{-s} \mathrm{~d} s,
$$

for all $z \neq 0$, and

$$
\Omega_{p, q}^{m, n}(s)=\frac{\prod_{j=1}^{m} \Gamma^{B_{i}}\left(b_{j}+\beta_{j} s\right) \prod_{j=1}^{n} \Gamma^{A_{j}}\left(1-a_{j}-\alpha_{j} s\right)}{\prod_{j=n+1}^{p} \Gamma^{A_{i}}\left(a_{j}+\alpha_{j} s\right) \prod_{j=m+1}^{q} \Gamma^{B_{j}}\left(1-b_{j}-\beta_{j} s\right)} .
$$


If $\Delta>0$, the integral (1) converges when $|\arg z|<\frac{1}{2} \Delta$; where

$$
\Delta=\sum_{j=1}^{m} B_{j} \beta_{j}-\sum_{j=m+1}^{q} B_{j} \beta_{j}+\sum_{j=1}^{n} A_{j} \alpha_{j}-\sum_{j=n+1}^{p} A_{j} \alpha_{j} .
$$

The generalized polynomials defined by Srivastava [23], is given in the following manner:

$$
\begin{aligned}
& S_{N_{1}, \cdots, N_{s}}^{M_{1}, \cdots, M_{s}}\left[y_{1}, \cdots, y_{s}\right]=\sum_{K_{1}=0}^{\left[N_{1} / M_{1}\right]} \cdots \sum_{K_{s}=0}^{\left[N_{s} / M_{s}\right]} \frac{\left(-N_{1}\right)_{M_{1} K_{1}}}{K_{1} !} \cdots \frac{\left(-N_{s}\right)_{M_{s} K_{s}}}{K_{s} !} \\
& \times A\left[N_{1}, K_{1} ; \cdots ; N_{s}, K_{s}\right] y_{1}^{K_{1}} \cdots y_{s}^{K_{s}}
\end{aligned}
$$

where $M_{1}, \cdots, M_{s}$ are arbitrary positive integers; and the coefficients $A\left[N_{1}, K_{1} ; \cdots ; N_{s}, K_{s}\right]$ are arbitrary constants (real or complex).

The multivariable $I$-function defined by Prathima et al. [14] (see also, [8]) is a extension of the multivariable $H$-function $[5 ; 6 ; 22 ; 24]$. It is defined in term of multiple Mellin-Barnes type integral, given by

$$
\begin{aligned}
& I\left(z_{1}, \cdots, z_{r}\right)=I_{p, q: p_{1}, q_{1} ; \cdots ; p_{r}, q_{r}}^{0, n: m_{1}, n_{1} ; \cdots ; m_{r}, n_{r}}\left[\begin{array}{c|c}
z_{1} \\
\cdot & \left(a_{j} ; \alpha_{j}^{(1)}, \cdots, \alpha_{j}^{(r)} ; A_{j}\right)_{1, p}: \\
\cdot & \left(b_{j} ; \beta_{j}^{(1)}, \cdots, \beta_{j}^{(r)} ; B_{j}\right)_{1, q}: \\
z_{r} &
\end{array}\right. \\
& \left(c_{j}^{(1)}, \gamma_{j}^{(1)} ; C_{j}^{(1)}\right)_{1, p_{1}} ; \cdots ;\left(c_{j}^{(r)}, \gamma_{j}^{(r)} ; C_{j}^{(r)}\right)_{1, p_{r}} \\
& \left.\left(d_{j}^{(1)}, \delta_{j}^{(1)} ; D_{j}^{(1)}\right)_{1, q_{1}} ; \cdots ;\left(d_{j}^{(r)}, \delta_{j}^{(r)} ; D_{j}^{(r)}\right)_{1, q_{r}}\right] \\
& =\frac{1}{(2 \pi \omega)^{r}} \int_{L_{1}} \cdots \int_{L_{r}} \phi\left(s_{1}, \cdots, s_{r}\right)\left\{\prod_{i=1}^{r} \theta_{i}\left(s_{i}\right) z_{i}^{s_{i}}\right\} \mathrm{d} s_{1} \cdots \mathrm{d} s_{r},
\end{aligned}
$$

where $\omega=\sqrt{-1} ; \phi\left(s_{1}, \cdots, s_{r}\right)$ and $\theta_{i}\left(s_{i}\right)$ for $i=1, \cdots, r$ are given as follows

$$
\begin{gathered}
\phi\left(s_{1}, \cdots, s_{r}\right)=\frac{\prod_{j=1}^{n} \Gamma^{A_{j}}\left(1-a j+\sum_{i=1}^{r} \alpha_{j}^{(i)} s_{j}\right)}{\prod_{j=n+1}^{p} \Gamma^{A_{j}}\left(a_{j}-\sum_{i=1}^{r} \alpha_{j}^{(i)} s_{j}\right) \prod_{j=1}^{q} \Gamma^{B_{j}}\left(1-b j+\sum_{i=1}^{r} \beta_{j}^{(i)} s_{j}\right)}, \\
\theta_{i}\left(s_{i}\right)=\frac{\prod_{j=1}^{n_{i}} \Gamma^{C_{j}^{(i)}}\left(1-c_{j}^{(i)}+\gamma_{j}^{(i)} s_{i}\right) \prod_{j=1}^{m_{i}} \Gamma^{D_{j}^{(i)}}\left(d_{j}^{(i)}-\delta_{j}^{(i)} s_{i}\right)}{\prod_{j=n_{i}+1}^{p_{i}} \Gamma^{C_{j}^{(i)}}\left(c_{j}^{(i)}-\gamma_{j}^{(i)} s_{i}\right) \prod_{j=m_{i}+1}^{q_{i}} \Gamma^{D_{j}^{(i)}}\left(1-d_{j}^{(i)}+\delta_{j}^{(i)} s_{i}\right)},
\end{gathered}
$$


for more details, reader can refer to recent work given by Prathima et al. [14].

Following the result of Braaksma [3], the $I$-function of $r$ variables is analytic if

$$
U_{i}=\sum_{j=1}^{p} A_{j} \alpha_{j}^{(i)}-\sum_{j=1}^{q} B_{j} \beta_{j}^{(i)}+\sum_{j=1}^{p_{i}} C_{j}^{(i)} \gamma_{j}^{(i)}-\sum_{j=1}^{q_{i}} D_{j}^{(i)} \delta_{j}^{(i)} \leqslant 0 \quad(i=1, \ldots, r) .
$$

The integral (6) converges absolutely if

$$
\begin{gathered}
\left|\arg \left(z_{k}\right)\right|<\frac{1}{2} \Delta_{k} \pi \quad(k=1, \ldots, r), \text { where } \\
\Delta_{k}=-\sum_{j=n+1}^{p} A_{j} \alpha_{j}^{(k)}-\sum_{j=1}^{q} B_{j} \beta_{j}^{(k)}+\sum_{j=1}^{m_{k}} D_{j}^{(k)} \delta_{j}^{(k)} \\
-\sum_{j=m_{k}+1}^{q_{k}} D_{j}^{(k)} \delta_{j}^{(k)}+\sum_{j=1}^{n_{k}} C_{j}^{(k)} \gamma_{j}^{(k)}-\sum_{j=n_{k}+1}^{p_{k}} C_{j}^{(k)} \gamma_{j}^{(k)}>0 .
\end{gathered}
$$

The complex numbers $z_{i}$ are not zero. Throughout this paper, we assume the existence and absolute convergence conditions of the multivariable $I$-function.

We will note

$$
\begin{gathered}
A=\left(a_{j} ; \alpha_{j}^{(1)}, \ldots, \alpha_{j}^{(r)} ; A_{j}\right)_{1, p} \\
B=\left(b_{j} ; \beta_{j}^{(1)}, \ldots, \beta_{j}^{(r)} ; B_{j}\right)_{1, q} \\
C=\left(c_{j}^{(1)}, \gamma_{j}^{(1)} ; C_{j}^{(1)}\right)_{1, p_{1}} ; \ldots ;\left(c_{j}^{(r)}, \gamma_{j}^{(r)} ; C_{j}^{(r)}\right)_{1, p_{r}} . \\
D=\left(d_{j}^{(1)}, \delta_{j}^{(1)} ; D_{j}^{(1)}\right)_{1, q_{1}} ; \ldots ;\left(d_{j}^{(r)}, \delta_{j}^{(r)} ; D_{j}^{(r)}\right)_{1, q_{r}} .
\end{gathered}
$$

The Mellin transform of $f(x)$ will be denoted by $M[f(x)]$ or $F(s)$. If $p$ and $y$ are real, we write $s=p^{-1}+i y$. If $p \geqslant 1, f(x) \in L_{p}(0, \infty)$, then for $p=1$ we have

$$
\begin{gathered}
M[f(x)]=F(s)=\int_{0}^{\infty} x^{s-1} f(x) \mathrm{d} x \text { and } f(x)=\frac{1}{2 i \pi} \int_{L} F(s) x^{-s} \mathrm{~d} s . \\
\text { For } p>0, M[f(x)]=F(s)=\text { l.i.m. } \int_{1 / x}^{x} x^{s-1} f(x) \mathrm{d} x,
\end{gathered}
$$

where, l.i.m. denotes the usual limit in the mean for $L_{p}-$ spaces. 


\section{DEFINITIONS}

The pair of new extended fractional integral operators are defined by the following equations:

$$
\begin{aligned}
& Q_{\gamma_{n}}^{\alpha, \beta}[f(x)] \\
& =t x^{-\alpha-t \beta-1} \int_{0}^{x} y^{\alpha}\left(x^{t}-y^{t}\right)^{\beta} I\left(\begin{array}{c|c}
\gamma_{1} v_{1} & A: C \\
\cdot & \\
\cdot & \\
\cdot & B: D
\end{array}\right) \prod_{j=1}^{k} \vec{I}_{M_{j}^{\prime \prime}, N_{j}^{\prime \prime}}^{M_{j}^{\prime}, N_{j}^{\prime}}\left[z_{j}\left(\frac{y^{t}}{x^{t}}\right)^{a_{j}}\left(1-\frac{y^{t}}{x^{t}}\right)^{b_{j}}\right] \\
& \times \prod_{j=1}^{r} S_{N_{1}^{M_{1}} M_{1}^{(j)}, \ldots, N_{s}^{(j)}}^{(j)}\left(\begin{array}{c}
z_{1}^{(j)}\left(\frac{y^{t}}{x^{t}}\right)^{g_{1}^{(j)}}\left(1-\frac{y^{t}}{x^{t}}\right)^{h_{1}^{(j)}} \\
\cdot \\
\cdot \\
\cdot \\
z_{s}^{(j)}\left(\frac{y^{t}}{x^{t}}\right)^{g_{s}^{(j)}}\left(1-\frac{y^{t}}{x^{t}}\right)^{h_{s}^{(j)}}
\end{array}\right) \psi\left(\frac{y^{t}}{x^{t}}\right) f(y) \mathrm{d} y .
\end{aligned}
$$

$$
\begin{aligned}
& R_{\gamma_{n}}^{\rho, \beta}[f(x)] \\
& =t x^{\rho} \int_{x}^{\infty} y^{-\rho-t \beta-1}\left(y^{t}-x^{t}\right)^{\beta} I\left(\begin{array}{c|c}
\gamma_{1} \mu_{1} & A: C \\
\cdot & \cdot \\
\cdot & \cdot \\
\cdot & B: D \\
\gamma_{n} \mu_{n}
\end{array}\right) \prod_{j=1}^{k} \bar{I}_{M_{j}^{\prime \prime}, N_{j}^{\prime \prime}}^{M_{j}^{\prime}, N^{\prime}}\left[z_{j}\left(\frac{x^{t}}{y^{t}}\right)^{a_{j}}\left(1-\frac{x^{t}}{y^{t}}\right)^{b_{j}}\right] \\
& \times \prod_{j=1}^{r} S_{N_{1}^{(j)}, \cdots, N_{s}^{(j)}}^{M_{s}^{(j)}}\left(\begin{array}{c}
z_{1}^{(j)}\left(\frac{x^{t}}{y^{t}}\right)^{g_{1}^{(j)}}\left(1-\frac{x^{t}}{y^{t}}\right)^{h_{1}^{(j)}} \\
\cdot \\
\cdot \\
\cdot \\
z_{s}^{(j)}\left(\frac{x^{t}}{y^{t}}\right)^{g_{s}^{(j)}}\left(1-\frac{x^{t}}{y^{t}}\right)^{h_{s}^{(j)}}
\end{array}\right) \psi\left(\frac{x^{t}}{y^{t}}\right) f(y) \mathrm{d} y,
\end{aligned}
$$

where, $v_{i}=\left(\frac{y^{t}}{x^{t}}\right)^{u_{i}}\left(1-\frac{y^{t}}{x^{t}}\right)^{v_{i}}, \mu_{i}=\left(\frac{x^{t}}{y^{t}}\right)^{u_{i}}\left(1-\frac{x^{t}}{y^{t}}\right)^{v_{i}}$ and $t, u_{i}, v_{i}, g_{i}^{(j)}, h_{i}^{(j)}, a_{j}, b_{j}$ are positive numbers.

The kernels $\psi\left(\frac{y^{t}}{x^{t}}\right)$ and $\psi\left(\frac{x^{t}}{y^{t}}\right)$ appearing in (17) and (18) respectively, are assumed to be continuous functions such the integrals make sense for wide classes of function 
$f(x)$.

The conditions for existence of these operators are as follows:

(a) $f(x) \in L_{p}(0, \infty)$, (b) $1 \leqslant p, q<\infty, p^{-1}+q^{-1}=1$.

(c) $\Re\left(\alpha+t a_{j} \frac{b_{j^{\prime} j}}{\beta_{j^{\prime} j}}\right)+t \sum_{i=1}^{n} u_{i} \min _{1 \leqslant j \leqslant m_{i}} \Re\left(\frac{d_{j}^{(i)}}{\bar{\delta}_{j}^{(i)}}\right)>-q^{-1}$.

(d) $\Re\left(\beta+t b_{j} \frac{b_{j^{\prime} j}}{\beta_{j^{\prime} j}}\right)+t \sum_{i=1}^{n} v_{i} \min _{1 \leqslant j \leqslant m_{i}} \Re\left(\frac{d_{j}^{(i)}}{\bar{\delta}_{j}^{(i)}}\right)>-q^{-1}$.

(e) $\Re\left(\rho+t a_{j} \frac{b_{j^{\prime} j}}{\beta_{j^{\prime} j}}\right)+t \sum_{i=1}^{n} u_{i} \min _{1 \leqslant j \leqslant m_{i}} \Re\left(\frac{d_{j}^{(i)}}{\bar{\delta}_{j}^{(i)}}\right)>-p^{-1}$ where $j=1 \ldots, k ; j^{\prime}=1, \ldots, M_{j}^{\prime}$.

Condition $(a)$ ensures that both operators defined in (17) and (18) exist and belong to. These operators are extensions of fractional integral operators defined and studied by several authors like Erdélyi [7], Love [13], Saigo et al. [17], Saxena and Kiryakova [18], Saxena and Kumbhat [20; 21], and etc.

\section{MAIN RESULTS}

THEOREM 1. If $f(x) \in L_{p}(0, \infty), 1 \leqslant p \leqslant 2$; or $f(x) \in L_{p}(0, \infty), p>2$, also following conditions satisfied:

$$
\begin{aligned}
& p^{-1}+q^{-1}=1, \\
& \Re\left(\alpha+t a_{j} \frac{b_{j^{\prime} j}}{\beta_{j^{\prime} j}}\right)+t \sum_{i=1}^{n} u_{i} \min _{1 \leqslant j \leqslant m_{i}} \Re\left(\frac{d_{j}^{(i)}}{\bar{\delta}_{j}^{(i)}}\right)>-q^{-1}, \\
& \Re\left(\beta+t b_{j} \frac{b_{j^{\prime} j}}{\beta_{j^{\prime} j}}\right)+t \sum_{i=1}^{n} v_{i} \min _{1 \leqslant j \leqslant m_{i}} \Re\left(\frac{d_{j}^{(i)}}{\bar{\delta}_{j}^{(i)}}\right)>-q^{-1},
\end{aligned}
$$

and the integrals present are absolutely convergent, then

$$
M\left\{Q_{\gamma_{n}}^{\alpha, \beta}[f(x)]\right\}=M\{f(x)\} R_{\gamma_{n}}^{\alpha-s+1, \beta}[1],
$$

where $M_{p}(0, \infty)$ stands for the class of all functions $f(x)$ of $L_{p}(0, \infty)$ with $p>2$, which are inverse Mellin-transforms of the function of $L_{p}(-\infty, \infty)$. 
PROOF. By making Mellin transform of (17), we get

$$
\begin{aligned}
& M\left\{Q_{\gamma_{n}}^{\alpha, \beta}[f(x)]\right\}=\int_{0}^{\infty} x^{s-1}\left\{t x^{-\alpha-t \beta-1} \int_{0}^{x} y^{\alpha}\left(x^{t}-y^{t}\right)^{\beta} I\left(\begin{array}{c|c}
\gamma_{1} v_{1} & A: C \\
\cdot & \\
\cdot & \cdot \\
\cdot & B: D \\
\gamma_{n} v_{n} &
\end{array}\right)\right. \\
& \left.\prod_{j=1}^{k} \bar{I}_{M_{j}^{\prime \prime}, N_{j}^{\prime \prime}}^{M_{j}^{\prime}, z_{j}^{\prime}}\left[z_{j}\left(\frac{y^{t}}{x^{t}}\right)^{a_{j}}\left(1-\frac{y^{t}}{x^{t}}\right)^{b_{j}}\right] S_{N_{1}^{(j)}, \ldots, N_{s}^{(j)}}^{M_{j}^{(j)}, \ldots, M_{s}^{(j)}}\left(\begin{array}{c}
z_{1}^{(j)}\left(\frac{y^{t}}{x^{t}}\right)^{g_{1}^{(j)}}\left(1-\frac{y^{t}}{x^{t}}\right)^{h_{1}^{(j)}} \\
\cdot \\
\cdot \\
\cdot \\
z_{s}^{(j)}\left(\frac{y^{t}}{x^{t}}\right)^{g_{s}^{(j)}}\left(1-\frac{y^{t}}{x^{t}}\right)^{h_{s}^{(j)}}
\end{array}\right) f(y) \mathrm{d} y\right\} \mathrm{d} x
\end{aligned}
$$

On interchanging the order of integration, which is permissible under the conditions, the result (19) follows in view of (18).

THEOREM 2. If $f(x) \in L_{p}(0, \infty), 1 \leqslant p \leqslant 2$; or $f(x) \in L_{p}(0, \infty), p>2$, also satisfied following conditions:

$p^{-1}+q^{-1}=1$

$\Re\left(\beta+t b_{j} \frac{b_{j^{\prime} j}}{\beta_{j^{\prime} j}}\right)+t \sum_{i=1}^{n} v_{i} \min _{1 \leqslant j \leqslant m_{i}} \Re\left(\frac{d_{j}^{(i)}}{\bar{\delta}_{j}^{(i)}}\right)>-q^{-1}$,

$\Re\left(\rho+t a_{j} \frac{b_{j^{\prime} j}}{\beta_{j^{\prime} j}}\right)+t \sum_{i=1}^{n} u_{i} \min _{1 \leqslant j \leqslant m_{i}} \Re\left(\frac{d_{j}^{(i)}}{\bar{\delta}_{j}^{(i)}}\right)>-p^{-1}$,

and the integrals present are absolutely convergent, then

$$
M\left\{R_{\gamma_{n}}^{\rho, \beta}[f(x)]\right\}=M\{f(x)\} Q_{\gamma_{n}}^{\rho+s-1, \beta}[1],
$$

where $M_{p}(0, \infty)$ stands for the class of all functions $f(x)$ of $L_{p}(0, \infty)$ with $p>2$, which are inverse Mellin-transforms of the function of $L_{p}(-\infty, \infty)$. 
PROOF. By making Mellin transform of (18), we get

$$
\begin{aligned}
& M\left\{R_{\gamma_{n}}^{\rho, \beta}[f(x)]\right\}=\int_{0}^{\infty} x^{s-1}\left\{t x^{\rho} \int_{x}^{\infty} y^{-\rho-t \beta-1}\left(y^{t}-x^{t}\right)^{\beta} I\left(\begin{array}{c|c}
\gamma_{1} \mu_{1} & A: C \\
\cdot & \\
\cdot & \cdot \\
\cdot & B: D \\
\gamma_{n} \mu_{n} &
\end{array}\right)\right. \\
& \times \prod_{j=1}^{k} \vec{I}_{M_{j}^{\prime \prime}, N_{j}^{\prime \prime}}^{M_{j}^{\prime}, N_{j}^{\prime}}\left[z_{j}\left(\frac{x^{t}}{y^{t}}\right)^{a_{j}}\left(1-\frac{x^{t}}{y^{t}}\right)^{b_{j}}\right]
\end{aligned}
$$

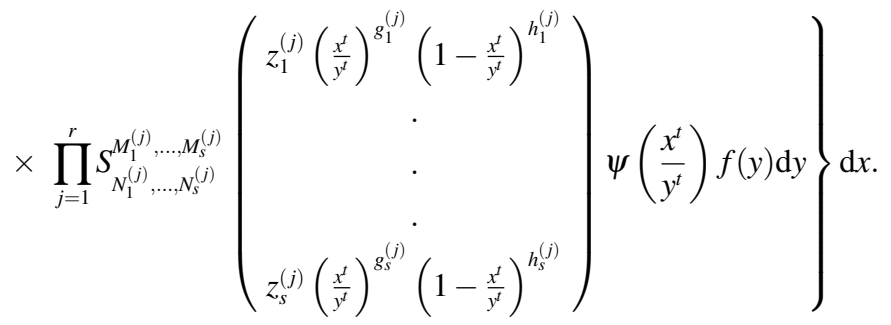

THEOREM 3. If $f(x) \in L_{p}(0, \infty), v(x) \in L_{p}(0, \infty)$, also satisfied following conditions:

$p^{-1}+q^{-1}=1$

$$
\begin{aligned}
& \Re\left(\alpha+t a_{j} \frac{b_{j^{\prime} j}}{\beta_{j^{\prime} j}}\right)+t \sum_{i=1}^{n} u_{i} \min _{1 \leqslant j \leqslant m_{i}} \Re\left(\frac{d_{j}^{(i)}}{\bar{\delta}_{j}^{(i)}}\right)>\max \left\{p^{-1}, q^{-1}\right\}, \\
& \Re\left(\beta+t b_{j} \frac{b_{j^{\prime} j}}{\beta_{j^{\prime} j}}\right)+t \sum_{i=1}^{n} v_{i} \min _{1 \leqslant j \leqslant m_{i}} \Re\left(\frac{d_{j}^{(i)}}{\bar{\delta}_{j}^{(i)}}\right)>0,
\end{aligned}
$$

and the integrals present are absolutely convergent, then

$$
\int_{0}^{\infty} v(x) Q_{\gamma_{n}}^{\alpha, \beta}[f(x)] \mathrm{d} x=\int_{0}^{\infty} f(x) R_{\gamma_{n}}^{\alpha, \beta}[v(x)] \mathrm{d} x .
$$

PRoOF. The result of (23) can be obtained in view of (17) and (18). 


\section{INVERSION FORMULAS}

THEOREM 4. If $f(x) \in L_{p}(0, \infty), 1 \leqslant p \leqslant 2$; or $f(x) \in L_{p}(0, \infty), p>2$, also following conditions satisfied:

$p^{-1}+q^{-1}=1$

$\Re\left(\alpha+t a_{j} \frac{b_{j^{\prime} j}}{\beta_{j^{\prime} j}}\right)+t \sum_{i=1}^{n} u_{i} \min _{1 \leqslant j \leqslant m_{i}} \Re\left(\frac{d_{j}^{(i)}}{\bar{\delta}_{j}^{(i)}}\right)>-q^{-1}$,

$\Re\left(\beta+t b_{j} \frac{b_{j^{\prime} j}}{\beta_{j^{\prime} j}}\right)+t \sum_{i=1}^{n} v_{i} \min _{1 \leqslant j \leqslant m_{i}} \Re\left(\frac{d_{j}^{(i)}}{\bar{\delta}_{j}^{(i)}}\right)>-q^{-1}$,

and the integrals present are absolutely convergent, also we have

$$
Q_{\gamma_{n}}^{\alpha, \beta}[f(x)]=v(x),
$$

then

$$
f(x)=\int_{0}^{\infty} y^{-1}[v(y)]\left[h\left(\frac{x}{y}\right)\right] \mathrm{d} y,
$$

where

$$
\begin{gathered}
h(x)=\frac{1}{2 i \pi} \int_{c-i \infty}^{c+i \infty} y^{-1} \frac{x^{-s}}{R(s)} \mathrm{d} s, \\
R(s)=R_{\gamma_{n}}^{\alpha-s+1, \beta}[1] .
\end{gathered}
$$

PROOF. On taking Mellin transform of (24) and then applying Theorem 1, we get

$$
M\{f(x)\}=\frac{M\{v(x)\}}{R(s)}
$$

which on inverting leads to

$$
f(x)=\frac{1}{2 i \pi} \int_{c-i \infty}^{c+i \infty} x^{-s} \frac{M\{v(x)\}}{R(s)} \mathrm{d} s=\frac{1}{2 i \pi} \int_{c-i \infty}^{c+i \infty} \frac{x^{-s}}{R(s)}\left\{\int_{0}^{\infty}[v(y)] \mathrm{d} y\right\} \mathrm{d} s .
$$

Interchanging the order of integration, we obtain

$$
f(x)=\int_{0}^{\infty} \frac{v(y)}{y}\left\{\frac{1}{2 i \pi} \int_{c-i \infty}^{c+i \infty}\left(\frac{x}{y}\right)^{s} \frac{1}{R(s)} \mathrm{d} s\right\} \mathrm{d} y .
$$

Now in view of (26), we obtain the desired result (25). 
THEOREM 5. If $f(x) \in L_{p}(0, \infty), 1 \leqslant p \leqslant 2$; or $f(x) \in L_{p}(0, \infty), p>2$, also satisfied following conditions:

$p^{-1}+q^{-1}=1$

$\Re\left(\beta+t b_{j} \frac{b_{j^{\prime} j}}{\beta_{j^{\prime} j}}\right)+t \sum_{i=1}^{n} v_{i} \min _{1 \leqslant j \leqslant m_{i}} \Re\left(\frac{d_{j}^{(i)}}{\bar{\delta}_{j}^{(i)}}\right)>-q^{-1}$,

$\Re\left(\rho+t a_{j} \frac{b_{j^{\prime} j}}{\beta_{j^{\prime} j}}\right)+t \sum_{i=1}^{n} u_{i} \min _{1 \leqslant j \leqslant m_{i}} \Re\left(\frac{d_{j}^{(i)}}{\bar{\delta}_{j}^{(i)}}\right)>-p^{-1}$,

and the integrals present are absolutely convergent, also we have

$$
R_{\gamma_{n}}^{\rho, \beta}[f(x)]=w(x),
$$

then

$$
f(x)=\int_{0}^{\infty} y^{-1}[w(y)]\left[g\left(\frac{x}{y}\right)\right] \mathrm{d} y
$$

where

$$
\begin{gathered}
g(x)=\frac{1}{2 i \pi} \int_{c-i \infty}^{c+i \infty} y^{-1} \frac{x^{-s}}{T(s)} \mathrm{d} s, \\
T(s)=Q_{\gamma_{n}}^{\rho+s-1, \beta}[1] .
\end{gathered}
$$

PROOF. On taking Mellin transform of (28) and then applying Theorem 2, we get

$$
M\{f(x)\}=\frac{M\{w(x)\}}{T(s)}
$$

which on inverting leads to

$$
f(x)=\frac{1}{2 i \pi} \int_{c-i \infty}^{c+i \infty} x^{-s} \frac{M\{w(x)\}}{T(s)} \mathrm{d} s=\frac{1}{2 i \pi} \int_{c-i \infty}^{c+i \infty} \frac{x^{-s}}{T(s)}\left\{\int_{0}^{\infty}[w(y)] \mathrm{d} y\right\} \mathrm{d} s .
$$

Interchanging the order of integration, we obtain

$$
f(x)=\int_{0}^{\infty} \frac{w(y)}{y}\left\{\frac{1}{2 i \pi} \int_{c-i \infty}^{c+i \infty}\left(\frac{x}{y}\right)^{s} \frac{1}{T(s)} \mathrm{d} s\right\} \mathrm{d} y .
$$

Now in view of (30), we obtain the desired result (29). 


\section{GENERAL PROPERTIES}

The properties given below are consequences of the definitions (17) and (18).

$$
\begin{gathered}
x^{-1} Q_{\gamma_{n}}^{\alpha, \beta}\left[\frac{1}{x} f\left(\frac{1}{x}\right)\right]=R_{\gamma_{n}}^{\alpha, \beta}[f(x)], \\
x^{-1} R_{\gamma_{n}}^{\rho, \beta}\left[\frac{1}{x} f\left(\frac{1}{x}\right)\right]=Q_{\gamma_{n}}^{\rho, \beta}[f(x)], \\
x^{\mu} Q_{\gamma_{n}}^{\alpha, \beta}[f(x)]=Q_{\gamma_{n}}^{\alpha-\mu, \beta}\left[x^{\mu} f(x)\right], \\
x^{\mu} R_{\gamma_{n}}^{\rho, \beta}[f(x)]=R_{\gamma_{n}}^{\rho+\mu, \beta}\left[x^{\mu} f(x)\right] .
\end{gathered}
$$

The properties given below express the homogeneity of operator $Q$ and $R$ respectively.

$$
\begin{aligned}
& \text { If } Q_{\gamma_{n}}^{\alpha, \beta}[f(x)]=v(x) \text { then } Q_{\gamma_{n}}^{\alpha, \beta}[f(c x)]=v(c x), \\
& \text { If } R_{\gamma_{n}}^{\rho, \beta}[f(x)]=w(x) \text { then } R_{\gamma_{n}}^{\rho, \beta}[f(c x)]=w(c x) .
\end{aligned}
$$

\section{MULTIVARIABLE $H$-FUNCTION}

If $A_{j}=B_{j}=C_{j}^{(i)}=D_{j}^{(i)}=1$, the multivariable $I$-function defined by Prathima et al. [14] reduces to multivariable $H$-function. We obtain the two following operators:

$$
\begin{aligned}
& Q_{\gamma_{n}}^{\alpha, \beta}[f(x)] \\
& =t x^{-\alpha-t \beta-1} \int_{0}^{x} y^{\alpha}\left(x^{t}-y^{t}\right)^{\beta} H\left(\begin{array}{c|c}
\gamma_{1} v_{1} & A: C \\
\cdot & \\
\cdot & \cdot \\
\cdot & B: D
\end{array}\right) \prod_{j=1}^{k} \bar{I}_{M_{j}^{\prime \prime}, N_{j}^{\prime \prime}}^{M^{\prime}, N^{\prime}}\left[z_{j}\left(\frac{y^{t}}{x^{t}}\right)^{a_{j}}\left(1-\frac{y^{t}}{x^{t}}\right)^{b_{j}}\right] \\
& \times \prod_{j=1}^{r} S_{N_{1}^{(j)}, \ldots, N_{s}^{(j)}}^{(j)}\left(\begin{array}{c}
z_{1}^{(j)}\left(\frac{y^{t}}{x^{t}}\right)^{g_{1}^{(j)}}\left(1-\frac{y^{t}}{x^{t}}\right)^{h_{1}^{(j)}} \\
\cdot \\
\cdot \\
\cdot \\
z_{s}^{(j)}\left(\frac{y^{t}}{x^{t}}\right)^{g_{s}^{(j)}}\left(1-\frac{y^{t}}{x^{t}}\right)^{h_{s}^{(j)}}
\end{array}\right) \psi\left(\frac{y^{t}}{x^{t}}\right) f(y) \mathrm{d} y,
\end{aligned}
$$

under the same notations and conditions that (17) with $A_{j}=B_{j}=C_{j}^{(i)}=D_{j}^{(i)}=1$. 


$$
\begin{aligned}
& R_{\gamma_{n}}^{\rho, \beta}[f(x)] \\
& =t x^{\rho} \int_{x}^{\infty} y^{-\rho-t \beta-1}\left(y^{t}-x^{t}\right)^{\beta} H\left(\begin{array}{c|c}
\gamma_{1} \mu_{1} & A: C \\
\cdot & \cdot \\
\cdot & \cdot \\
\cdot & B: D
\end{array}\right) \prod_{j=1}^{k} \vec{I}_{M_{j}^{\prime \prime}, N_{j}^{\prime \prime}}^{M_{j}^{\prime}, N^{\prime}}\left[z_{j}\left(\frac{x^{t}}{y^{t}}\right)^{a_{j}}\left(1-\frac{x^{t}}{y^{t}}\right)^{b_{j}}\right] \\
& \times \prod_{j=1}^{r} S_{N_{1}^{(j)}, \ldots, N_{s}^{(j)}}^{M_{1}^{(j)}, \ldots, M_{s}^{(j)}}\left(\begin{array}{c}
z_{1}^{(j)}\left(\frac{x^{t}}{y^{t}}\right)^{g_{1}^{(j)}}\left(1-\frac{x^{t}}{y^{t}}\right)^{h_{1}^{(j)}} \\
\cdot \\
\cdot \\
\cdot \\
z_{s}^{(j)}\left(\frac{x^{t}}{y^{t}}\right)^{g_{s}^{(j)}}\left(1-\frac{x^{t}}{y^{t}}\right)^{h_{s}^{(j)}}
\end{array}\right) \psi\left(\frac{x^{t}}{y^{t}}\right) f(y) \mathrm{d} y
\end{aligned}
$$

under the same notations and conditions that (18) with $A_{j}=B_{j}=C_{j}^{(i)}=D_{j}^{(i)}=1$. We obtain the same theorems and properties concerning these operators.

\section{CONCLUSION}

The functions involved in the results established in this paper are unified and general nature, hence a large number of known results lying in the literature follows as special cases. Further, on suitable specifications of the parameters involved, numerous new results involving simpler functions may also be obtained.

\section{DISCLOSURE STATEMENT}

No potential conflict of interest was reported by the authors. 


\section{REFERENCES}

F. Y. Ayant, An integral associated with the Aleph-functions of several variables, Int. J. Math. Trends Tech. 31(3) (2016), 142-154.

D. Baleanu, D. Kumar and S. D. Purohit, Generalized fractional integrals of product of two $H$-functions and a general class of polynomials, Int. J. Comput. Math. 93(8) (2016), 1320-1329.

B.L.J. Braaksma, Asymptotic expansions and analytic continuations for a class of Barnes integrals, Compos. Math. 15 (1964), 239-341.

V.B.L. Chaurasia and A. Srivastava, A unified approach to fractional calculus pertaining to $H$-functions, Soochow J. Math. 33(2) (2007), 211-221.

J. Choi, J. Daiya, D. Kumar and R. K. Saxena, Fractional differentiation of the product of Appell function $F_{3}$ and multivariable $H$-function, Commun. Korean Math. Soc., 31(1) (2016), 115-129.

J. Daiya, J. Ram and D. Kumar, The multivariable $H$-function and the general class of Srivastava polynomials involving the generalized Mellin-Barnes contour integrals, FILOMAT 30(6) (2016), 14571464.

A. Erdélyi, On some functional transformations, Univ. Politec. Torino, Rend. Sem. Mat. 10 (1950-51), 217234.

D. Kumar and F.Y. Ayant, Some double integrals involving multivariable I-function, Acta Universitatis Apulensis 58(2) (2019), 35-43.

D. Kumar, F.Y. Ayant and J. Choi, Application of product of the multivariable $A$-function and the multivariable Srivastava polynomials, East Asian Math. J. 34(3) (2018), 295-303.

D. Kumar and J. Daiya, Fractional calculus pertaining to generalized $H$-functions, Glob. J. Sci. Front. Res. (F) 14(3) (2014), 25-35.

D. Kumar, R.K. Gupta, B.S. Shaktawat and J. Choi, Generalized fractional calculus formulas involving the product of Aleph-function and Srivastava polynomials, Proc. Jangjeon Math. Soc. 20(4) (2017), 701717.

D. Kumar, S. D. Purohit and J. Choi, Generalized fractional integrals involving product of multivariable $H$-function and a general class of polynomials, J. Nonlinear Sci. Appl. 9 (2016), 8-21.

E.R. Love, Some integral equations involving hypergeometric functions, Proc. Edinb. Math. Soc. 15 (3) (1967), 169-198.

J. Prathima, V. Nambisan and S. K. Kurumujji, A study of I-function of several complex variables, Int. J. Engrg. Math. 2014 (2014), 1-12.

J. Ram and D. Kumar, Generalized fractional integration involving Appell hypergeometric function of the product of two $H$-functions, Vijanana Parishad Anusandhan Patrika 54(3) (2011), 33-43.

A.K. Rathie, A new generalization of generalized hypergeometric function, Le Matematiche 52(2) (1997), $297-310$ 
M. Saigo, R.K. Saxena and J. Ram, On the fractional calculus operator associated with the $H$-function, Ganita Sandesh 6(1) (1992), 36-47.

R.K. Saxena and V.S. Kiryakova, On relation between the two-dimensional $H$-transforms in terms of Erdélyi-Kober operators, Math. Balkanica 6 (1992), 133-140.

R.K. Saxena and D. Kumar, Generalized fractional calculus of the Aleph-function involving a general class of polynomials, Acta Math. Sci. Ser. B Engl. Ed. 35(5) (2015), 1095-1110.

R.K. Saxena and R.K. Kumbhat, Fractional integration operators of two variables, Proc. Indian Acad. Sci. Math. Sci. 78 (1973), 177-186.

R.K. Saxena and R.K. Kumbhat, Some properties of generalized Kober operators, Vijnana Parishad Anusandhan Patrika 18 (1975), 139-150.

R.K. Saxena, J. Ram and D.L. Suthar, Unified fractional derivative formulas for the multivariable $H$ function, Vijnana Parishad Anusandhan Patrika 49(2) (2006), 159-175.

H.M. Srivastava, A multilinear generating function for the Konhauser set of biorthogonal polynomials suggested by Laguerre polynomial, Pacific J. Math. 177 (1985), 183-191.

H.M. Srivastava and R. Panda, Some bilateral generating functions for a class of generalized hypergeometric polynomials, J. Reine Angew. Math. 283/284 (1976), 265-274.

\title{
Dinesh Kumar
}

Department of Applied Sciences, College of Agriculture, Sumerpur-Pali, Agriculture University of Jodhpur, Jodhpur 342304, India email: dinesh_dino03@yahoo.com

\author{
Frédéric Ayant \\ Collége Jean L'herminier, \\ Allée des Nymphéas, 83500 La Seyne-sur-Mer, France \\ Six-Fours-les-Plages 83140, Department of Var, France \\ email: fredericayant@gmail.com
}

